\title{
ELECTROMAGNETIC SIMULATIONS AND PROPERTIES OF THE FUNDAMENTAL POWER COUPLERS FOR THE SNS SUPERCONDUCTING CAVITIES*
}

\author{
Y. Kang ${ }^{\dagger}$, S. Kim, M. Doleans, Spallation Neutron Source, Oak Ridge, TN 37830, USA \\ I. E. Campisi, M. Stirbet, P. Kneisel, G. Ciovati, G. Wu, TJNAF, Newport News, VA 23606, USA \\ P. Ylä-Oijala, Rolf Nevanlinna Institute, University of Helsinki, Finland
}

\begin{abstract}
The Spallation Neutron Source (SNS) makes use of superconducting cavities for the acceleration of negative $\mathrm{H}$ ions in the main linac. Two types of 6-cell Niobium cavities are used in the superconducting portion of the linac: $33 \beta=0.61$ cavities and $48 \beta=0.81$ cavities. Each cavity is powered via a coaxial fundamental power coupler (FPC) of a simple yet robust design. The electromagnetic design of the main components of that coupler has been modeled and some of those properties have been measured experimentally. Modeling includes impedance matching of the window and of the waveguide to coaxial doorknob transition; coupling of the coupler fields to the cavity fields; and multipacting behavior of the coaxial line and window. Various aspects of design, simulation, and testing on the coupler and cavity are presented.
\end{abstract}

\section{INTRODUCTION}

Coaxial Fundamental Power Couplers (FPC) with coaxial ceramic windows will be used with the superconducting 6-cell cavities in the SNS main linac [1]. The basic coupler design is derived, with slight modifications, from the one KEK developed for $508.8 \mathrm{MHz}$, due to its simplicity in design and dependability at high power operation [2]. For higher operating RF frequency in the SNS linac, the mechanical dimensions of the critical parts of the coupler have been scaled proportionately. The fundamental power couplers for the SNS superconducting linac cavities will have to satisfy the following specifications:

\section{Frequency: $\quad 805 \mathrm{MHz}$ \\ Peak power: $550 \mathrm{~kW}$ (SNS klystrons forward power) \\ Duty factor: $\quad 7.8 \%,(60 \mathrm{pps}, 1.3 \mathrm{msec}$ pulses $)$ \\ External Q: $\quad 7.3 \times 10^{5}(\beta=0.61), 7.0 \times 10^{5}(\beta=0.81)$}

The coupler includes the antenna, annular disk-type ceramic window, and the waveguide to coaxial transition. The RF properties of the coupler parts have been checked and assured by modeling in 3-D RF structure simulator codes. The cavity external Q-factor $\left(\mathrm{Q}_{\mathrm{ext}}\right)$ is determined by penetration of the coupling antenna in the beam pipe at one end of the cavity. The scaled window section was modeled with the HFSS code. The doorknob-type

\footnotetext{
* Supported by US DOE Contract No. DE-AC05-00OR22725

†kangyw@sns.gov
}

waveguide to coaxial transition was also simulated using HFSS to finalize the dimensions. A coaxial high voltage capacitor is formed at the junction of the coaxial innerconductor and the doorknob. The capacitor design is somewhat similar to the solution adopted for the KEKB coupler [3].

The multipacting characteristic of the coaxial coupler structure was computer-simulated and the result is discussed. The $\mathrm{Q}_{\text {ext }}$ 's of the 6-cell cavities $(\beta=0.61,0.81)$ have been predicted by modeling the coupler and the cavity in MAFIA 3-D code. Bench measurements were performed and the results were compared with the predictions.

\section{CERAMIC WINDOW MATCHING}

The coaxial window is a planar annular disk-type made of $95 \%$ alumina ceramic. The chokes at both sides of the ceramic window have the important role of improving the window matching. The depths of the chokes were optimized in the simulations to have lowest return loss and insertion loss.
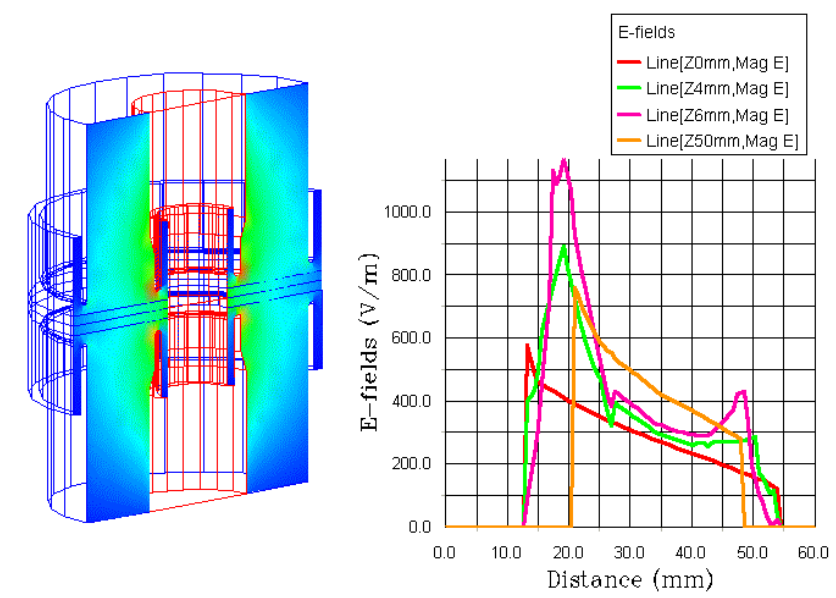

Figure 1: Half structure of 3-D model for the coaxial section of the ceramic window used in HFSS. Timeinstantaneous peak fields normalized to $1 \mathrm{~W}$ input are shown near the ceramic window at $\mathrm{z}=0,4,6,50 \mathrm{~mm}$.

Figure 1 shows the window structure modeled using HFSS. Shaded plot of the time-instantaneous electric field magnitude is shown with the structure. Radial distribution of the electric fields at various locations around the ceramic window is also shown. The results show that the highest field developed around the tips of the inductive 
chokes on the inner-conductor of the structure. Fundamental RF characteristics of the window at 805 $\mathrm{MHz}$ were comparable to those of the $508.8 \mathrm{MHz}$ design. The calculated values of the return loss are better than -50 $\mathrm{dB}$ and the insertion loss is around $0.005 \mathrm{~dB}$ at $805 \mathrm{MHz}$.

\section{DOOR KNOB TRANSITION}

\subsection{Simulations}

The waveguide to coaxial transition has a doorknobtype impedance matching structure. The transition alone must have good impedance matching to assure best performance when integrated with the ceramic window. Even a small mechanical change inside the structure can result in a significant change in the RF performance. Such disturbances are grooves and slits and rounded corners, etc. Details of the structure were included in the modeling. The transition is also computer simulated using the HFSS code. The doorknob height, diameter, and the distance between the doorknob and the short circuit were varied to find the optimum performance in terms of the return loss and the insertion loss. The waveguide to coaxial transition can have either a polygonal tapered short or a circular rounded short circuit at the end of the rectangular waveguide. Simulations were made for transitions with both short circuit shapes and the results showed that both types have similar RF performances. Manufacturing costs will most likely determine the choice between the two short circuit types.

The coaxial structure with the ceramic window and the doorknob waveguide transition have been integrated in a simulation. The structure used in the modeling and the electric-field distributions are shown in Figure 2. In the integrated structure the return loss at the waveguide input port is slightly degraded. In that Figure the shaded plot of time-instantaneous electric-field magnitude is shown together with the structure. Also, the time instantaneous electric-field vectors are shown along the coaxial structure on the plane of symmetry at $r=1 \mathrm{in}$.

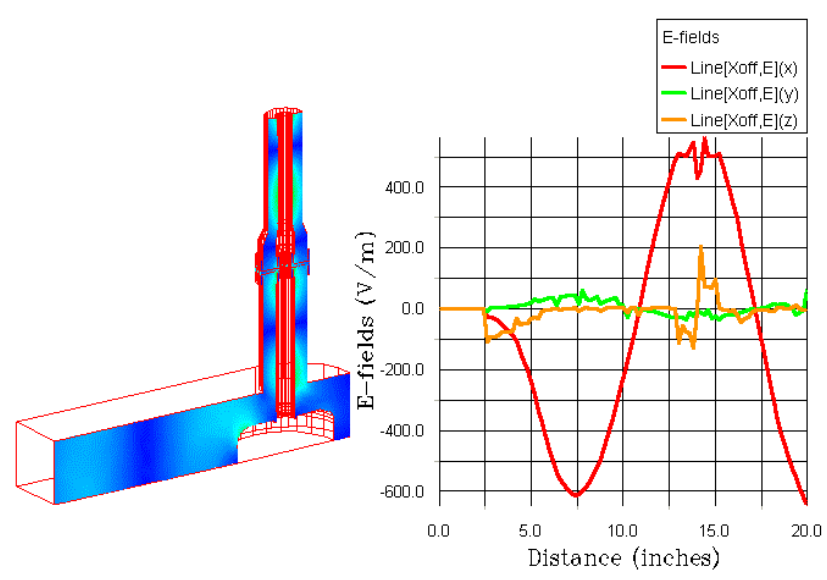

Figure 2: Half structure used in the HFSS modeling: the doorknob transition is integrated with the coaxial window.

\subsection{Biasing capacitor design}

A high-voltage capacitor is implemented at the junction of the center conductor and the doorknob transition to control the multipacting during high power conditioning and operation. The capacitor is designed to have a quarter wavelength electrically at $805 \mathrm{MHz}$ in the insulating dielectric medium. For its high dielectric strength and high temperature characteristics, a $0.125 \mathrm{~mm}$ thick Kapton sheet has been used as the insulator. The outer conductor of the capacitor becomes a part of the doorknob and has axial cuts to be able to have good compression on the Kapton dielectric for a stable capacitor structure. The corner of the doorknob-capacitor junction is rounded with a 0.25 in. radius.

\section{COUPLER EXTERNAL Q}

\subsection{Simulations}

A computer study of $\mathrm{Q}_{\mathrm{ext}}$ has been done using the scheme that is adequate for high Q system [4]. The fiveparameter space in Figure 3 has been explored for the reference geometry. Among them, the inner conductor tip position has the strongest effect on the coupling between the cavity and the power coupler. As shown in Figure 5, about a $20 \mathrm{~mm}$ displacement results in one order of magnitude variation on the $\mathrm{Q}_{\mathrm{ext}}$ value. The required $\mathrm{Q}_{\mathrm{ext}} \mathrm{s}$ are $7.3 \times 10^{5}$ and $7.0 \times 10^{5}( \pm 20 \%)$ for medium and high beta cavities, respectively. The corresponding inner conductor tip position is about $9 \mathrm{~mm}(+2 \mathrm{~mm} /-1.5 \mathrm{~mm})$ into the beam pipe. Concerning the other imperfections such as field non-uniformity, mechanical tolerances etc., the inner conductor tip position accuracy should be \pm 1 $\mathrm{mm}$ or less to satisfy the $\mathrm{Q}_{\mathrm{ext}}$ requirement.
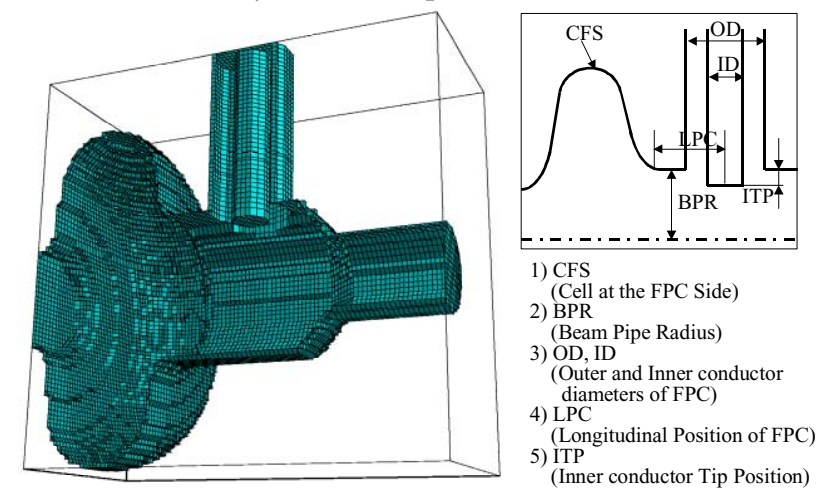

Figure 3. MAFIA 3-D model for the $\mathrm{Q}_{\mathrm{ext}}$ analysis and the five parameters that affect $\mathrm{Q}_{\mathrm{ext}}$

\subsection{Measurements.}

To measure the $\mathrm{Q}_{\mathrm{ext}}$ of the fundamental power coupler, an auxiliary antenna at the opposite end of the cavity has been used to excite the fundamental mode. The transmitted signal has been picked-up at the FPC. The measurement setup is shown in Figure 4. The length of the auxiliary antenna has to be such as there is enough coupling, to minimize measurements errors, and no 
perturbation in cavity's frequency and field profile. By measuring the $\mathrm{Q}$-loaded $\left(\mathrm{Q}_{\mathrm{L}}\right)$, the coupling factor $(\beta)$ of the auxiliary antenna, and the transmission coefficient $\left(\mathrm{S}_{21}\right)$ with a calibrated network analyzer, it is possible to determine the $\mathrm{Q}_{\mathrm{ext}}$ of the FPC according to the following formula:

$$
Q_{\text {ext }}=\frac{4 \beta \cdot Q_{L}}{(1+\beta)} 10^{\mid S_{21}[d B] / 10}
$$

The length of the center conductor has been changed using spacers and $\mathrm{Q}_{\mathrm{ext}}$ measurements for different tip penetrations have been done. The results are in good agreement with the calculated ones (Figure 5).

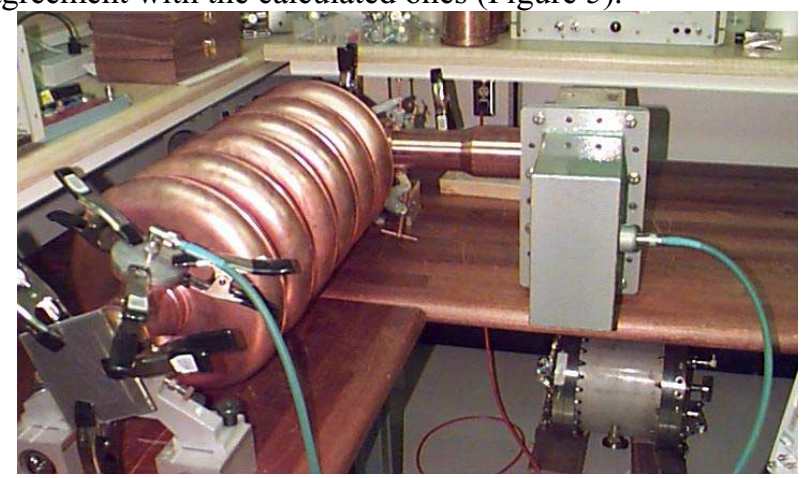

Figure 4: $\mathrm{Q}_{\mathrm{ext}}$ measurements setup.

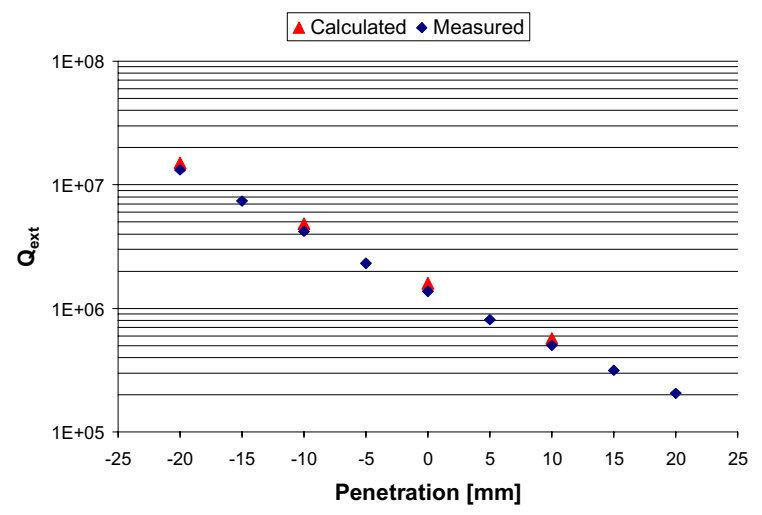

Figure 5: $Q_{\text {ext }}$ calculated and measured for the $\beta=0.61$ cavity.

\section{MULTIPACTING}

Simulations of the multipacting behavior of the SNS coupler were performed to predict the power levels at which this process might occur and to identify the locations where most multipacting would occur and which may prevent operation of this specific design. The details of the calculations are reported in [5].

The analysis has been performed first in the purely coaxial lines. Since these dimensions have been scaled directly by frequency from KEK's coupler, a similar behavior was expected since the multipacting power levels scale as $(f d)^{4} Z$. Multipacting bands are predicted to exist in both sizes of coaxial lines (outer conductors 76 and $96 \mathrm{~mm}$ ). The calculations have been done both in standing wave mode and in mixed waves (travelling waves with varying reflection coefficient).
Whereas the calculations in the coaxial line are common to any coaxial design, more specific simulations have been conducted to study the behavior of the coaxial taper and of the window assembly. Multipacting activities have been predicted to exist at the tapered transition for power levels of 320,420 and $480 \mathrm{~kW}$ in various mixed waves conditions. Similarly, multipacting between the window surface and the output conductor can exist at 140 and $795 \mathrm{~kW}$.

All of the above multipacting activities can be disrupted, after RF conditioning, by judicious use of the biasing voltage (calculations with biasing voltage have not been conducted at this time). Simulations of multipacting activities at the windows' matching chokes show that some multipacting can occur between the window and the choke, and within the inner choke itself. These cannot be affected by voltage biasing. Multipacting levels of 220, 250 and $300 \mathrm{~kW}$ have been predicted for these phenomena for a purely imaginary reflection coefficient. Figure 6 shows the case with $250 \mathrm{~kW}$ power.
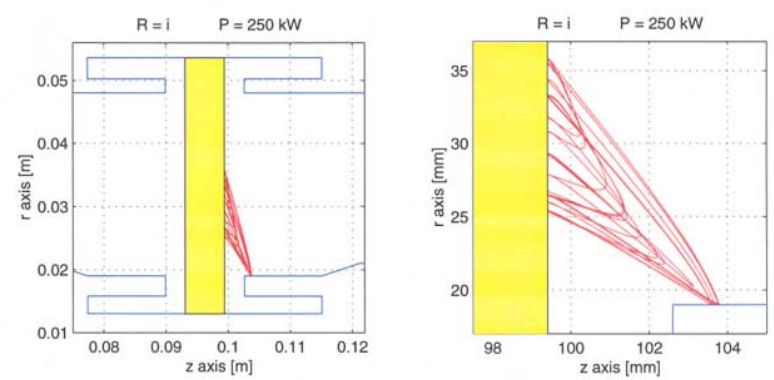

Figure 6: Multipacting trajectories between the window and the matching choke.

\section{CONCLUSIONS}

Several types of electromagnetic simulations have been performed on various components of the SNS superconducting cavity coaxial fundamental power coupler. The results of the simulations have been extremely useful in predicting and correcting potential problems. Given the extremely short time available for the development of the coupler, the availability of simulations has been invaluable to the success of the coupler development effort.

\section{REFERENCES}

[1] I. E. Campisi et al., "The Fundamental Power Coupler Prototype for the Spallation Neutron Source (SNS) Superconducting Cavities," PAC 2001, Chicago, IL, June 2001.

[2] S. Mitsunobu, et al., "High Power Input Coupler for KEKB SC Cavity," $9^{\text {th }}$ Workshop on RF Superconductivity, Santa Fe, November 1999.

[3] S. Mitsunobu et al., "Status and Development of Superconducting Cavity for KEKB," PAC97, Vancouver, BC, May 1997.

[4] P. Balleyguier, "External Q Studies for APT SCCavity Couplers," LINAC98, Chicago IL, August 1998.

[5] P. Ylä-Oijala and M. Ukkola, "Multipacting simulations on the coaxial SNS coupler," Internal Report, Rolf Nevanlinna Institute, Helsinki, November 2000. 